\title{
Determinants of Sputum Ordering for Tuberculosis Diagnosis in People Living with HIV in the Greater Accra Region, Ghana
}

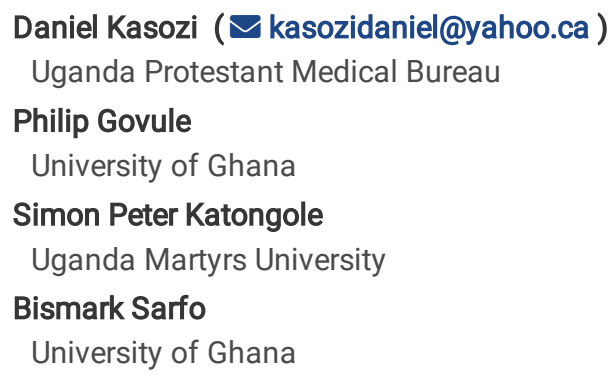




\section{Abstract}

\section{Background}

Tuberculosis (TB) remains a significant public health concern, and a leading cause of ill-health and death globally. More so, People living with HIV have been shown to carry an increased risk of developing TB with an estimated one-third of deaths in this population. The World Health Organization recommends systematic and routine screening of PLHIV for TB on every clinic visit and further testing using sputum for those with a positive TB screen test. Not all PLHIV with a positive TB screen test in Ghana are further tested for TB using sputum and the factors for this are not well understood. This study assessed factors associated with sputum ordering for TB diagnosis in PLHIV who were screened positive for TB in three hospitals providing HIV care and treatment services in the Greater Accra region of Ghana.

\section{Methods}

Mixed method study performed at three purposively selected hospitals providing HIV care and treatment services in the Greater Accra region. The study involved a cross sectional review of patients' charts and in-depth interviews with health workers involved in the care and treatment of PLHIV. Quantitative data was analyzed using STATA version 15. Chi square test was used for bivariate analysis. Logistic regression was used for multivariate analysis. $\mathrm{P} \leq 0.05$ was considered statistically significant. Inductive thematic analysis was used to determine emerging themes from the interviews. The major themes were represented with representative quotations.

\section{Results}

Four hundred (400) patient charts were reviewed of which $67.7 \%$ were female with median age of 39 (IQR 31-49). TB screening was recorded in $78 \%(95 \% \mathrm{Cl} 73.6,82.0)$ of the patients of whom ninety-two (92) patients had a positive TB screen test. Only $53(57.6 \%)$ who had a positive screen test had sputum ordered for further TB testing. In the multivariate analysis, patient general appearance described as abnormal $(O R=3.05$, $p=0.036)$, having more than one TB symptom $(O R=3.42, p=0.028)$ and presence of an alternative presumptive diagnosis $(O R=0.34, p=0.023)$ were associated with having a sputum test ordered. High patient numbers, inability to produce sputum, unwillingness of the not so sick patients to provide sputum and the costs associated with chest X-ray were perceived as the challenges to further testing for TB.

\section{Conclusion}

TB screening in PLHIV is still lower than recommended and almost half of PLHIV with a positive TB screen test did not have a sputum test documented. Sputum testing was likely to be done in patients with an abnormal general appearance and more than one TB symptom and unlikely in those with an alternative presumptive diagnosis. High workload, costs of TB tests, lack of training for health workers and inability to produce sputum by patients were the barriers to sputum testing highlighted by the health workers.

\section{Background}

In populations considered with a high HIV prevalence, tuberculosis (TB) remains a public health burden despite the progress made towards its control, and it is among the top causes of morbidity and mortality. Each year, an estimated 10 million people develop TB, a number that has remained stable over the years [1]. TB is the top-most cause of mortality from a single infectious agent, ranking above HIV/AIDS. In 2018, an estimated 1.2 million TB deaths occurred in HIV uninfected people and 251,000 deaths in HIV infected people [1]. This unacceptably high mortality due to TB underscores the importance of early TB direction and treatment especially in people living with HIV (PLHIV).

Through destruction of the CD4-T lymphocytes and thus impairing cellular immune function, HIV is the most recognized risk factor for progression from TB infection to active TB disease [3]. The risk of developing active TB disease is 20 to 37 times greater in HIV infected persons than in HIV uninfected persons and it is the leading cause of mortality in people living with HIV [4].

In many high burden TB/HIV countries including Ghana, many of the estimated new TB cases are un-notifiable [5]. Only $64 \%$ of the estimated 10 million new TB cases in HIV uninfected persons and $51 \%$ of the estimated 0.9 million new TB cases in HIV infected persons were reported in 2017 [5]. According to the World Health Organization (WHO), this may be due to underreporting or under diagnosis of TB; either patients do not or cannot access health care, or they are missed when they finally do access healthcare [1].

Among PLHIV and other most at-risk populations, WHO recommends systematic screening of all patients at every clinic visit and further evaluation of those with a positive TB symptom screen test with Gene-Xpert-MTB/RIF or Acid Fast Bacillus (AFB) microscopy where the former is not available [6]. HIV infected adults and adolescents are considered to have a positive TB screen test if they have any of the four symptoms of current cough, fever, weight loss or night sweats. Children at least one year of age living with HIV are considered to have a positive TB screen test if they have any of the four symptoms of current cough, fever, poor weight gain and history of contact with a TB patient [7]. 
Whereas this recommendation was made for TB screening among PLHIV, only a small proportion of PLHIV with a positive TB symptom screen test are referred for sputum examination for TB detection even in high burden TB/HIV settings(8). In a study in Uganda among HIV infected patients, only $13.2 \%$ of the patients with a positive TB screen test had a sputum order documented [8]. In another study conducted in Uganda, $54.9 \%$ of HIV infected patients with a positive TB symptom screen test were referred for sputum smear examination [9].

Ghana has a high TB/HIV burden, considered among the top 30 countries with a high TB/HIV burden [1]. In 2018, with an estimated incident rate of 148 per 100,000 population, there was an estimated 44,000 incident cases of TB [10]. The incidence rate of TB among PLHIV was 29 per 100,000 translating into 8,600 TB cases among PLHIV [10]. More men than women developed TB in the same year; 26,000 vs 18,000 new TB cases. In the same period, there were 11,000 deaths due to TB among HIV negative patients and 4,800 deaths due to TB among PLHIV [10]. Of estimated 44,000 new TB cases in 2017, only 14,289 (32.3\%) were reported [10]. This low TB case detection negates all efforts in place to end TB by the year 2050. The low TB detection in both HIV infected and uninfected people has been singled out as one of the challenges affecting TB control in Ghana [12]. The low TB case detection may partly be due to the fact that patients with a positive TB screen test don't get further evaluation to determine their definitive TB status. Only 27.5\% PLHIV with a positive TB symptom screen test had a documented sputum order for Acid Fast Bacillus (AFB) smear microscopy. The proportion only increased slightly to 50.7\% after an audit and feedback intervention [13] . This study assessed the factors associated with sputum ordering in PLHIV with a positive TB symptom screen test in the Greater Accra region of Ghana.

\section{Methods}

\section{Study site and population}

The study was conducted in three purposively selected (based on HIV infected patients registered) public hospitals providing HIV care and treatment in the Greater Accra region. These were: Greater Accra regional hospital, Tema general hospital and La general hospital. Greater Accra is one of the ten administrative regions of Ghana, occupying a total land surface of 3,254 square kilometers. With a total population of $4,010,054$ in 2010, it is the second most populated region in Ghana [11]. The study population was PLHIV who came to hospital between April and June 2019 and health care providers involved in screening and evaluation of TB in PLHIV.

\section{Study Design}

We conducted a concurrent mixed method study. The quantitative component involved a cross sectional retrospective chart review in which records of HIV infected patients who came for health care between April and June 2019 were reviewed. The quarter April to June was purposively selected so that the study results represent current practice. The qualitative component involved in-depth interviews with the health care providers involved in screening and testing for TB in PLHIV.

\section{Sample size and Sampling procedures}

The sample size for the quantitative component was calculated using the Leslie Kish 1994 formula:

$$
n=\frac{Z^{2} P Q}{\delta^{2}}
$$

Where: $\mathrm{Z}$ is the normal standard deviate at $95 \%$ level of confidence; $\mathrm{P}$ is the estimated prevalence of the problem under investigation; $\mathrm{Q}=1-\mathrm{P} ; \delta$ is the precision; Using $\mathrm{P}=0.507$ [10] and precision of $5 \%$, gives a minimum sample of 385; At each hospital, charts of patients who came for health care between April and June 2019 were obtained. Using disproportionate random sampling (the number of randomly selected patient charts from each study site was independent of the total number of patients registered at the site), 133 patient charts were selected at study site. Health care workers for the in-depth interviews were purposively selected depending on their role in screening and evaluating PLHIV for TB.

\section{Data Analysis}

Quantitative data was analyzed using StataIC version 15.0 (Stata Corporation, College Station, TX, USA). Descriptive characteristics of the categorical variables were summarized as frequencies, proportions and median for the continuous variables. At bivariate analysis, the Chi square test was used to determine association between the outcome variable and various independent variables. Where the expected frequencies were less than 5, the Fisher's exact test was used instead. Multivariate logistic regression was used to determine factors that are independently associated with sputum ordering. Backward elimination method was used to construct the multiple logistic regression model 
using a threshold of 0.2 to eliminate variables from the model. The likelihood ratio test for nested models was used to test the null hypothesis that the automatically selected multiple logistic regression model was a better fit for the data compared to the manually constructed model.

Variable with a p-value of $\leq 0.05$ were considered significant. Inductive thematic analysis was used for Qualitative data to determine emerging themes. The main themes were illustrated with representative quotations.

\section{Ethics Consideration}

Ethical clearance for the study (Approval No: GHS-ERC068/02/19) was obtained from the Ghana Health Service Ethical Review Board (GHS$\mathrm{ERC})$.

\section{Limitations}

The main limitation of the study was incomplete data records which resulted into dropping a number of variables. Data on current WHO stage (an indicator of disease severity) could not be collected since patients were staged only at enrollment. Furthermore, data on CD4 cell count and/or viral load could also not be collected because this data was not available.

\section{Results}

\section{Baseline and Clinical Characteristics of Patients}

In total, 400 patient charts were reviewed. Of these, 271 (67.8\%) were females, majority (29.7\%) were aged 35-44 years and 319 (79.8\%) were continuing clients (Table 1).

Table 1

Baseline and clinical characteristics of patients, $\mathrm{N}=400$

\begin{tabular}{|lll|}
\hline Characteristic & Category & No. (\%) \\
\hline Age category & $<15$ & $19(4.8)$ \\
& $15-24$ & $15(3.8)$ \\
& $25-34$ & $102(25.5)$ \\
& $35-44$ & $119(29.7)$ \\
& $45-54$ & $90(22.5)$ \\
& $\geq 55$ & $55(13.7)$ \\
\hline Sex & Female & $271(67.7)$ \\
\hline Visit type & Male & $129(32.3)$ \\
\hline & New & $81(20.2)$ \\
\hline WHO stage at enrollment & I & $319(79.8)$ \\
\hline & II & $126(31.5)$ \\
\hline & III & $134(33.5)$ \\
\hline IV & $33(8.3)$ \\
\hline
\end{tabular}

\section{Prevalence of TB symptoms, TB Screening and Sputum Ordering}

Of the 400 patients, $312(78.0 \%, 95 \% \mathrm{Cl} 73.6,82.0)$ were screened for TB. While of these 312 patients who were screened for TB, $92(29.5 \%)$ had a positive TB screen test and 53 (57.6\%) had a sputum order documented (Fig. 1).

Of the 92 patients with a positive TB screen test, majority (27.2\%) were aged $45-54$ years, 48 (52.2\%) were female and $42(45.6 \%)$ were new patients (Table 2). The most common TB symptom was cough, where more than half of the patients had cough (Fig. 2). 


\section{Factors Associated with Sputum Ordering in Patients with a positive TB Screen Test}

In the bivariate analysis, only patients' general appearance on the last visit, documented as abnormal $\left(X^{2}=9.45, p=0.002\right)$, having more than one (1) TB symptom $\left(X^{2}=8.17, p=0.004\right)$ and presence of a presumptive alternative diagnosis $\left(X^{2}=8.99, p=0.003\right)$, were significantly associated with sputum ordering for patients with a positive TB screen test (Table 2).

\section{Univariable logistic regression analysis results}

Before adjustment, patients with an abnormal appearance (OR $\left.\mathrm{O}^{\mathrm{u}}, 4.40 ; 95 \% \mathrm{Cl}, 1.65-11.72\right)$, and those with more than one symptom (OR ${ }^{\mathrm{u}}$, 4.22; $95 \% \mathrm{Cl}, 1.51-11.76)$, were significantly associated with higher odds of sputum order. Patients with abnormal appearance or having more than one symptom were over 4 times more likely to have a sputum order than those whose general appearance was normal and those having only one TB symptom. However, sputum order reduced by $73 \%\left(\mathrm{OR}^{\mathrm{u}}, 0.27 ; 95 \% \mathrm{Cl}, 0.11-0.65\right)$, for patients with a documented alternative diagnosis when compared to those in whom no alternative diagnosis was documented (Table 2).

\section{Multivariable logistic regression analysis results}

After adjustment, the odds of sputum ordering in patients whose general appearance was noted to be abnormal $\left(0 \mathrm{R}^{\mathrm{a}}=3.05 ; 95 \% \mathrm{Cl}, 1.07-\right.$ $1.67, p=0.036)$ were 3.05 times the odds of sputum ordering in patients whose condition was described as normal. Similarly, patients with more than one symptom were over three times more likely to have a sputum order, compared to those with one symptom $\left(\mathrm{OR}^{\mathrm{a}}=3.42 ; 95 \% \mathrm{Cl}\right.$, $1.15-$ $10.21, p=0.028$ ) were significantly associated with sputum ordering for patients with positive TB screening test. The odds of sputum ordering in patients who had an alternative presumptive diagnosis $\left(\mathrm{OR}^{\mathrm{a}}=0.36 ; 95 \% \mathrm{Cl}, 0.13-0.86, \mathrm{p}=0.023\right)$ were reduced by $66 \%$ when compared to those with no such diagnosis and this was significant, (Table 2).

Table 2

Bivariate and Multivariate analysis of factors associated with sputum ordering, $\mathrm{N}=92$ 
Sputum order

\begin{tabular}{|c|c|c|c|c|c|c|c|c|}
\hline Factor & Present, $\mathrm{n}(\%)$ & Absent, $\mathrm{n}(\%)$ & $x^{2}$ & $\mathrm{p}$-value & $\left(\mathrm{OR}^{\mathrm{u}}(95 \% \mathrm{Cl})\right.$ & $\mathrm{p}$-value & $\mathrm{OR}^{\mathrm{a}}(95 \% \mathrm{Cl})$ & $\mathrm{p}$-value \\
\hline \multicolumn{9}{|l|}{ Age category } \\
\hline$<15$ & $2(40)$ & $3(60)$ & $5.29^{\#}$ & 0.381 & 1 & & & \\
\hline $15-24$ & $0(0)$ & $3(100)$ & & & 0.00 & 0.999 & & \\
\hline $25-34$ & 19(63.3) & $11(36.7)$ & & & $2.59(0.37-17.98)$ & 0.335 & & \\
\hline $35-44$ & $12(54.5)$ & $10(45.5)$ & & & $1.80(0.25-12.98)$ & 0.560 & & \\
\hline $45-54$ & $16(64)$ & $9(36)$ & & & $2.66(0.37-19.10)$ & 0.328 & & \\
\hline$\geq 55$ & $4(57.1)$ & $3(43.9)$ & & & $2.00(0.19-20.61)$ & 0.560 & & \\
\hline \multicolumn{9}{|l|}{ Sex } \\
\hline Female & $27(55.1)$ & $22(44.9)$ & 0.07 & 0.783 & 1 & & & \\
\hline Male & $26(60.5)$ & 17(39.5) & & & $1.12(0.49-2.57)$ & 0.783 & & \\
\hline \multicolumn{9}{|l|}{ Visit type } \\
\hline New & $28(56.0)$ & $22(44.0)$ & 0.12 & 0.733 & 1 & & & \\
\hline Continuing & $25(59.5)$ & $17(40.5)$ & & & $1.16(0.51-2.65)$ & 0.733 & & \\
\hline \multicolumn{9}{|l|}{ Symptoms No. } \\
\hline 1 & $30(47.6)$ & $33(52.4)$ & 8.17 & 0.004 & 1 & & 1 & \\
\hline$>1$ & $23(79.3)$ & $6(20.7)$ & & & $4.22(1.51-11.76)$ & 0.006 & $3.42(1.15-10.21)$ & 0.028 \\
\hline \multicolumn{9}{|l|}{ Appearance } \\
\hline Normal & $27(45.8)$ & $32(54.2)$ & 9.45 & 0.002 & 1 & & 1 & \\
\hline Abnormal & $26(78.8)$ & $7(21.2)$ & & & $4.40(1.65-11.72)$ & 0.003 & $3.05(1.07-8.67)$ & 0.036 \\
\hline \multicolumn{9}{|l|}{ Alt diagnosis } \\
\hline Absent & $37(71.1)$ & 15(28.9) & 8.99 & 0.003 & 1 & & 1 & \\
\hline Present & $16(40)$ & $24(60)$ & & & $0.27(0.11-0.65)$ & 0.003 & $0.34(0.13-0.86)$ & 0.023 \\
\hline
\end{tabular}

\#=X² (fisher's exact test); Lrtest; $H_{0}$ : Automatically selected model better fit for the data. $X^{2}=0.82 p=0.84$. OR ${ }^{u}$ : unadjusted Odds Ratio; OR ${ }^{a}$ : adjusted Odds Ratio.

\section{Common Alternative Diagnoses and Prevalence of Chest X-ray Uptake}

The most common alternative diagnoses documented in the client folders were malaria and respiratory tract infections (RTI). Of the forty (40) patients that had an alternative diagnosis documented, half of them were reported to have RTI. The other diagnoses included meningitis, liver disease and weight loss (figure 3). Additionally, it may be essential to embrace the use of CXR as a screening tool for TB among PLHIV. Investigation of patients with CXR was very low, as only 3 out of $92(3.3 \%)$ of the patients with a positive screen test had a CXR order documented.

\section{Health worker Perceived Barriers to Sputum Ordering and Chest X-ray Use}

Four (4) out of the six (6) planned interviews were conducted. The two health workers were not willing to participate. All respondents were female. The health workers were interviewed for their perceptions about the potential barriers hindering screening of HIV infected patients for TB and further testing of those with a positive test. Some of the barriers are described below. 


\section{High Patient numbers}

High patient numbers resulting into a high workload was one of the barriers identified by the health workers as indicated in the statements below:

"The number of patients in this clinic is high. This sometimes hinders provision of quality services including TB screening and evaluation"

\section{Another health work said:}

"The clients here are so many. We cannot do much. As you know like elsewhere in Africa, the doctor/nurse to patient ratio is very low. However, we try to make sure that all patients get tested for TB at least twice a year."

\section{Inability by the Patients to produce sputum}

It was also mentioned several times that patients fail to produce sputum when they are asked to.

"Sometimes a patient comes, and you ask them to provide sputum, but he/she says I can't produce sputum, I have failed. So, in that case no test will be done".

Patients who are well will not be willing to provide sputum.

"Patients who are well do not want to provide sputum. They don't see the need for doing many tests. We cannot force them. However, those who are sick, try their best to provide the sputum for testing."

\section{Cost associated with Chest X-ray (CXR)}

The chest X-ray is one of the tests recommended by the GHS as part of the work up of PLHIV. However, its use was very low, and the cost associated with the $\mathrm{x}$-ray services was cited as the main barrier.

"Unlike sputum, chest X-ray is not free. Wherever the patients do x-rays they have to pay. This limits the uptake of the service as part of TB work up."Said one of the health workers.

Related to cost, another health worker had this to say:

"Although the chest X-ray is covered under the National Health Insurance scheme, patients have to pay money for the radiologist report to accompany the $x$-ray. This also makes the $x$-rays services unaffordable for most of the patients."

\section{Lack of integration of TB services into HIV care and treatment services}

From the interviews, it was apparent that there is lack of integration of TB services into HIV care and treatment services as represented by the following quotation

"There is a chest clinic that takes care of TB patients where patients with TB are sent for treatment and further evaluation. Even TB medicines for TB/HIV co-infected patients are not provided here, but at the chest clinic."

\section{Infrequent trainings to improve/build capacity of health workers.}

Health workers also noted that the frequency of the trainings is low. This affects quality of care especially when new health workers come or policies change. The following quotations represent the issue of low training frequency

"Once in a blue moon, we have workshops. The DOTS coordinator organizes trainings once in a blue moon"

Affirmed by a quotation from another health worker who seemed to stress the need for the trainings with an additional reason being the need to fill knowledge gap which is noted to be limited particularly among students.

"Training are few here. We have not had any in the last three months. They should increase the number of trainings. Aside we the staff, we sometimes have students here whose knowledge may be limited"

Page 7/13 


\section{Discussion}

TB screening in PLHIV in this study was fairly high at 78\%, though lower than the required target of $100 \%$. The proportion of patients with a positive TB symptom screen test was $29.5 \%$ out of which $57.6 \%$ had evidence of a sputum order. At both bivariate and multivariate analysis, patient general appearance described as abnormal, having more than one TB symptom and presence of an alternative diagnosis were significantly associated with sputum ordering among patients with a positive TB symptom screen.

The results from this study are comparable to results from other studies that investigated TB screening in PLHIV. In South Africa, the prevalence of TB screening was 89.6\% [12]. In Kenya TB screening prevalence was 98\% [13] while in Uganda the prevalence of TB screening was 93\% [14]. In all the studies cited above, the TB screening rate was far higher than what was found in this study. This could be due to the fact that all the studies cited above assessed TB screening of new patients at enrollment in care. This study reviewed data from the most recent patient visit, in majority of whom, it was not the initial visit. High TB screening levels tend not to be sustained [13], since in that particular study TB screening had dropped from $89.6-71 \%$ (which is similar to the coverage in this study) by the end of twelve months.

The proportion of patients with at least one TB symptom (positive TB screen test) in this study was $29.5 \%$. This finding is comparable to findings from other studies where the proportion was found to be $8 \%$ [16], 14\% [13], 28\% [7], 37.4\% [11] and 62\% [14].

In this study, only slightly more than half (57.6\%) of all patients with a positive TB screen test had evidence of a sputum order for further evaluation of TB. This is very low compared to the desired target of $100 \%$ and given the high burden of TB/HIV in Ghana. It is possible that this low investigation rates contributes to the high annual mortality due to TB in PLHIV [9]. Low sputum ordering in PLHIV with a positive TB screen test has been reported in a number of studies. In Ghana, a study that evaluated the impact of feedback on TB suspicion and investigation in PLHIV among health care providers found that only $27.5 \%$ of all PLHIV with a positive TB screen test were referred for a sputum test. This proportion increased to only $50.7 \%$ after the feedback intervention [10]. In South Africa, sputum ordering in PLHIV with a positive screen test was 35\% [12] while in Botswana, sputum and/or CXR ordering rates were reported to be 35.3\% in PLHIV with a positive TB screen test [15]. In a study conducted in Uganda to evaluate TB case detection services in the general population considering persons with cough of $\geq 2$ weeks duration, only $21 \%$ of the patients were referred for sputum examination. The proportion only increased to $53 \%$ at the end of the intervention [16]. In a Malawian study, sputum ordering in TB suspects was at 48\% [17]. As mentioned earlier, the low number of persons with a positive TB screen test undergoing further evaluation for TB, results into missed opportunities with resultant increase in TB related mortality in PLHIV [1]. In a study that investigated missed TB opportunities among autopsy patients, TB was missed in $52 \%$ of the cases. The study also found that of the $52 \%$ of the cases in which TB was missed, chest symptoms had been missed in at most $39 \%$ of the cases, sputum ordering was missed in up to $85 \%$ of the cases and CXR in up to $38 \%$ of the cases [18].

There appears to be selective application of the guidelines for intensified TB case detection in PLHIV observed in this study. PLHIV with more than one TB symptom and those whose general appearance was described as abnormal, were over three times more likely to have a sputum order compared to patients with neither of the conditions. This may imply that health worker attitude to further investigate patients with a positive TB screen is influenced by the general condition or appearance of the patient. Other studies have also found similar findings in which patients who are considered to have advanced disease have a higher likelihood of being investigated further for TB. In Uganda, patients with WHO stage III or IV (indicator of more advanced disease) were more likely to have sputum order [14]. Men were more likely than women to be investigated by sputum [12,19]. It should be noted that those studies reported that men were more likely to have advanced disease (lower CD4 cells $/ \mu l)$ compared to women. It is therefore likely that men were more likely to have a sputum order because of the more advanced disease status. In this study, sex was not associated with sputum ordering. This may be because most of the study participants were ART experienced which was not the case in all other studies, where participants were ART naïve. The more the number of TB symptoms, the more advanced disease that patient is likely to have and hence the more the likelihood of being investigated. In this study, the number of TB symptoms was significantly associated with general appearance-an indicator of health status in this study $\left(X^{2}=4.63, p=0.031\right)$. Similar findings were reported from a study conducted in Uganda [14]. This study also found that patients with an alternative diagnosis had odds of a sputum order reduced by $66 \%$ compared to patients with no alternative diagnosis. This implies that where a health worker could find an alternative diagnosis (mainly malaria and upper respiratory tract infection in this study) for the patient's symptoms, the index of suspicion of TB was significantly lowered. Similar findings were reported in a study conducted in Uganda [14].

It has been postulated that this selective evaluation of patients with a positive TB screen test is likely to be due to fear of health workers to over burden the already challenged TB laboratories [10]. Whereas this may be true to some extent, the interviews conducted with the health workers in this study, revealed that the main reasons for this behavior was the inability of patients to produce sputum, low risk perception in some patients, high patient numbers that constrain the few available health workers leading to non-adherence to national guidelines and the prohibitive costs associated with some TB tests such as CXR. In South Africa, fewer patients than expected with a positive TB screen test were investigated with sputum partly due to high occurrence of unproductive cough [12]. In a study conducted in Uganda to understand health worker perspectives on barriers to quality TB services delivery, health workers reported high work load resulting in low staff motivation as part of the

Page $8 / 13$ 
barriers [20]. This high workload is also likely to compromise the quality of health education (group and individual) provided to patients leading to the low risk perception among some of the patients with the resultant reluctance to provide specimens for TB diagnostic work up. In addition, costs associated with TB diagnostic test were aslo among the barriers for TB diagnostic work up [20]. It has been reported that where TB diagnostic tests are free, the uptake is higher [21].

\section{Recommendations}

In order to address some of the challenges reported in this study, the following recommendations are provided:

(i). The National AIDS/STI Control Program (NACP) should expedite the roll-out of the differentiated models of service delivery to reduce the patient numbers seen on clinic days. This reduced workload will not only lessen practical and financial hardships on the side of the patients but will also reduce the burden on the health system and improve the quality of care.

(ii). NACP should carry out continuous on-job training and mentorship of health workers on the importance of screening for TB for all PLHIV on every clinic visit and further evaluation of those with a positive screen test regardless of their health status.

(iii). Ghana Health Service (GHS) should reduce the costs associated with CXR especially in PLHIV. The cost can be taken up by the NACP which is mandated by WHO to cater for TB logistics for PLHIV.

(iv). NACP should roll out sputum induction strategy to facilitate obtaining of sputum from patients who cannot produce sputum like those with a dry cough and young children. This roll out involves training provision to health workers and availing the necessary equipment.

\section{Conclussion}

TB screening in PLHIV is still lower than recommended and almost half of PLHIV with a positive TB screen test did not have a sputum test documented. Sputum testing was likely to be done in patients with an abnormal general appearance, more than one TB symptom and unlikely in those with an alternative presumptive diagnosis. High workload, high costs of TB tests, lack of training for health workers and inability to produce sputum by patients were the barriers to sputum testing highlighted by the health workers.

\section{Abbreviations}

AFB - Acid Fast Bacilli; AIDS - Acquired Immune Deficiency Syndrome; ART - Antiretroviral Treatment; CXR - Chest x-ray; GHS - Ghana Health Service; HIV - Human Immunodeficiency Virus; IGRA - Interferon Gamma Release Assay; LTBI - Latent TB Infection; MOH - Ministry of Health; MTB - Mycobacterium Tuberculosis; NACP - National AIDS/STI Control Program; NPV - Negative Predicative Value; NSS - Number Needed to Screen; OR ${ }^{\mathrm{a}}$ - adjusted Odds Ratio; OR ${ }^{\mathrm{u}}$ - Unadjusted Odds Ratio; PLHIV - People Living with HIV; PPV - Positive Predicative Value; RIF Rifampicin; SMM - Sputum Smear Microscopy; SSA - Sub Saharan Africa; TB - Tuberculosis; TST - Tuberculin Skin Test; WHO - World Health Organization.

\section{Declarations}

\section{Ethics Approval and Consent to Participate}

Ethical clearance for the study (Approval No: GHS-ERC068/02/19) was obtained from the Ghana Health Service Ethical Review Board (GHSERC). Written informed consent was obtained from participants taking part in the qualitative part of the study.

\section{Consent for publication}

Not applicable

\section{Availability of data and materials}

The datasets used and/or analyzed during the study are available from the corresponding author on reasonable request.

\section{Competing interests}




\section{Funding}

This study was elaborated based on the work of Daniel Evans Kasozi, a Master of Public Health (MPH) scholarship recipient and, used grants from the World Health Organization obtained through the Special Programme for Research and Training in Tropical Diseases (TDR). However, no support has been received from WHO/TDR or any other institution in regard to the preparation (design and analysis) and publication of this manuscript. Additionally, the authors are entirely responsible for all information expressed in this manuscript.

\section{Authors' Contributions}

DEK conceptualized and drafted the original paper. PG, SPK and BS reviewed the study design. DEK and PG undertook the statistical analysis. PG, SPK and BS reviewed the manuscript. All authors approved the final manuscript.

\section{Acknowledgement}

The authors acknowledge the people who helped in the collection and processing of the data and trancribing the qualitative data audio recordings, Medical directors and staff of all the participating health facilities.

\section{References}

1. WHO. Global TUBERCULOSIS REPORT [Internet]. 2019 [cited 2019 Dec 24]. Available from: http://apps.who.int/bookorders.

2. WHO. Global Tuberculosis Report 2018. World Heal Organ. 2018;1-134.

3. Aït-Khaled N, Alarcon E, Bissell K, Boillot F, Caminero JA, Chiang C-Y, et al. Isoniazid preventive therapy for people living with HIV: public health challenges and implementation issues [Internet]. Vol. 13, INT J TUBERC LUNG DIS. 2009 [cited 2018 Sep 26]. Available from: www.theunion.org]

4. WHO. Guidelines for intensified tuberculosis case-finding and isoniazid preventive therapy for people living with HIV in resource-constrained settings [Internet]. 2011 [cited 2018 Nov 14]. Available from:

http://apps.who.int/iris/bitstream/handle/10665/44472/9789241500708_eng.pdf;jsessionid=B4F09A2219384073A54C90BEB3CAA4C5? sequence $=1$

5. WHO. Global tuberculosis report [Internet]. 2018 [cited 2018 Oct 14]. Available from: http://apps.who.int/bookorders.

6. WHO. THE USE OF ANTIRETROVIRAL DRUGS FOR TREATING AND PREVENTING HIV INFECTION 2016 [Internet]. 2016 [cited 2018 Nov 10]. Available from:

http://apps.who.int/iris/bitstream/handle/10665/208825/9789241549684_eng.pdf;jsessionid=4DC07C1A3897EB91EEEC4A00C28C119B? sequence $=1$

7. WHO. Latent tuberculosis infection Updated and consolidated guidelines for programmatic management [Internet]. 2018 [cited 2018 Sep 14]. Available from: http://apps.who.int/iris/bitstream/handle/10665/260233/9789241550239-

eng.pdf;jsessionid=6B87A187FB6E519B2CE6CCC2B5D53EA7? sequence $=1$

8. Kerkhoff AD, Cattamanchi A. Diagnosis of HIV-Associated Tuberculosis. In: HIV and Tuberculosis [Internet]. Cham: Springer International Publishing; 2019 [cited 2019 Dec 25]. p. 127-59. Available from: http://link.springer.com/10.1007/978-3-030-29108-2_7

9. Roy M, Muyindike W, Vijayan T, Kanyesigye M, Bwana M, Wenger M, et al. Use of symptom screening and sputum microscopy testing for active tuberculosis case detection among HIV-infected patients in real-world clinical practice in Uganda. 2016 [cited 2018 Nov 5]; Available from: https://www.ncbi.nlm.nih.gov/pmc/articles/PMC5621516/pdf/nihms782789.pdf

10. Miller CR, Davis JL, Katamba A, Sserwanga A, Kakeeto S, Kizito F, et al. Sex disparities in tuberculosis suspect evaluation: a cross-sectional analysis in rural Uganda. Int J Tuberc Lung Dis [Internet]. 2013 Apr [cited 2018 Nov 2];17(4):480-5. Available from: http://www.ncbi.nlm.nih.gov/pubmed/23485382

11. WHO. Ghana Tuberculosis Profile [Internet]. 2019 [cited 2019 Dec 24]. Available from: https://extranet.who.int/sree/Reports? op=Replet\&name=\%2FWHO_HQ_Reports\%2FG2\%2FPROD\%2FEXT\%2FTBCountryProfile\&ISO2=GH\&LAN=EN\&outtype=pdf

12. WHO. GHANA TB PROFILE [Internet]. 2017 [cited 2018 Nov 14]. Available from: https://extranet.who.int/sree/Reports? op=Replet\&name=\%2FWHO_HQ_Reports\%2FG2\%2FPROD\%2FEXT\%2FTBCountryProfile\&ISO2=GH\&LAN=EN\&outtype=pdf

13. $\mathrm{MOH}$. The National Tuberculosis Health Sector Strategic Plan for Ghana; 2009-2013 [Internet]. 2009 [cited 2018 Nov 14]. Available from: http://www.tbonline.info/media/uploads/documents/national_tb_health_sector_strategic_plan_2009-2013_2.pdf

Page 10/13 
14. Bjerrum S, Bonsu F, Hanson-Nortey NN, Kenu E, Johansen IS, Andersen AB, et al. Tuberculosis screening in patients with HIV: use of audit and feedback to improve quality of care in Ghana. Glob Health Action [Internet]. 2016;9(1):32390. Available from:

https://www.tandfonline.com/doi/full/10.3402/gha.v9.32390

15. Ghana Statistical Service. 2010 Population \& Housing Census National Analytical Report. Ghana Stat Serv [Internet]. 2013;1-91. Available from: http://statsghana.gov.gh/docfiles/2010phc/National_Analytical_Report.pdf

16. Van Rie A, Clouse K, Hanrahan C, Selibas K, Sanne I, Williams S, et al. High uptake of systematic HIV counseling and testing and TB symptom screening at a primary care clinic in South Africa. PLoS One [Internet]. 2014 [cited 2019 Jun 19];9(9):e105428. Available from: http://www.ncbi.nlm.nih.gov/pubmed/25268851

17. Burmen B, Modi S, Cavanaugh JS, Muttai H, McCarthy KD, Alexander $\mathrm{H}$, et al. Tuberculosis screening outcomes for newly diagnosed persons living with HIV, Nyanza Province, Kenya, 2009. Int J Tuberc Lung Dis [Internet]. 2016 Jan [cited 2019 Jun 19];20(1):79-84. Available from: http://www.ncbi.nlm.nih.gov/pubmed/26688532

18. Bloss E, Makombe R, Kip E, Smit M, Chirenda J, Gammino VM, et al. Lessons learned during tuberculosis screening in public medical clinics in Francistown, Botswana. Int J Tuberc Lung Dis [Internet]. 2012 Aug [cited 2019 Jun 19];16(8):1030. Available from: http://www.ncbi.nlm.nih.gov/pubmed/22687289

19. Roy M, Muyindike W, Vijayan T, Kanyesigye M, Bwana M, Wenger M, et al. Implementation and Operational Research: Use of Symptom Screening and Sputum Microscopy Testing for Active Tuberculosis Case Detection Among HIV-Infected Patients in Real-World Clinical Practice in Uganda. J Acquir Immune Defic Syndr [Internet]. 2016 [cited 2018 Nov 2];72(5):e86-91. Available from: http://www.ncbi.nlm.nih.gov/pubmed/27159224

20. Davis J, Katamba A, Vasquez J, Crawford E, Sserwanga A, Kakeeto S, et al. Evaluating tuberculosis case detection via real-time monitoring of tuberculosis diagnostic services. Am J Respir Crit Care Med [Internet]. 2011 Aug 1 [cited 2019 Jun 19];184(3):362-7. Available from: http://www.ncbi.nlm.nih.gov/pubmed/21471088

21. Gawa LG, Reid T, Edginton ME, Van Lettow M, Joshua M, Harries AD. Public Health Action Diagnostic management and outcomes of pulmonary tuberculosis suspects admitted to a central hospital in Malawi Interna onal Union Against Tuberculosis and Lung Disease Health solu ons for the poor. 2011 [cited 2019 Jun 20]; Available from: http://dx.doi.org/10.5588/pha.11.0007

22. Field N, Murray J, Wong ML, Dowdeswell R, Dudumayo N, Rametsi L, et al. Missed opportunities in TB diagnosis: a TB Process-Based Performance Review tool to evaluate and improve clinical care. BMC Public Health [Internet]. 2011 Dec 22 [cited 2019 Jun 20];11(1):127. Available from: https://bmcpublichealth.biomedcentral.com/articles/10.1186/1471-2458-11-127

23. Cattamanchi A, Miller CR, Tapley A, Haguma P, Ochom E, Ackerman S, et al. Health worker perspectives on barriers to delivery of routine tuberculosis diagnostic evaluation services in Uganda: a qualitative study to guide clinic-based interventions. BMC Health Serv Res [Internet]. 2015 Dec 22 [cited 2018 Oct 15];15(1):10. Available from: http://bmchealthservres.biomedcentral.com/articles/10.1186/s12913014-0668-0

24. Fenner L, Ballif M, Graber C, Nhandu V, Dusingize JC, Cortes CP, et al. Tuberculosis in antiretroviral treatment programs in lower income countries: availability and use of diagnostics and screening. PLoS One [Internet]. 2013 [cited 2019 Jun 20];8(10):e77697. Available from: http://www.ncbi.nlm.nih.gov/pubmed/24147059

\section{Figures}




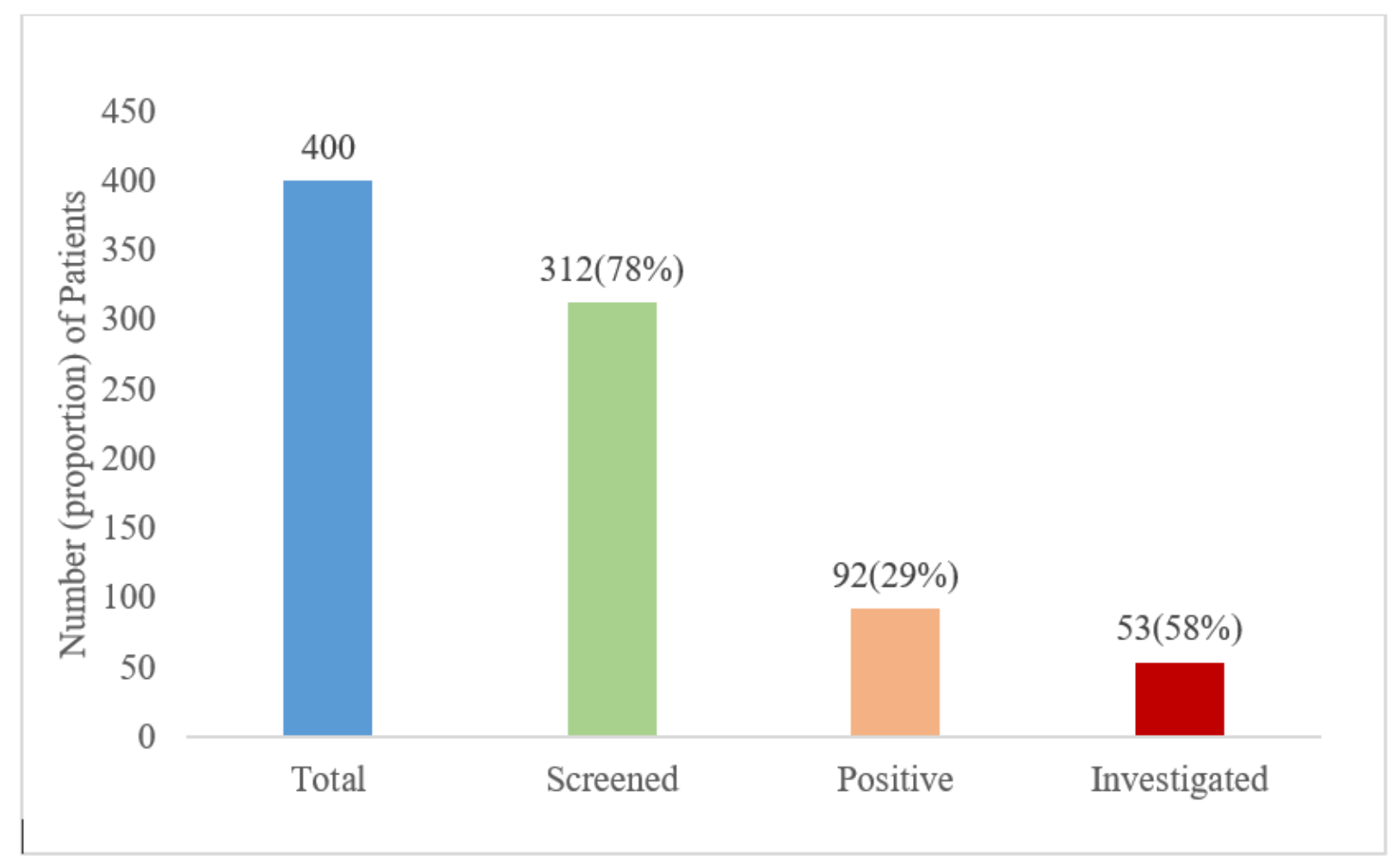

\section{Figure 1}

Drop-off of Patients along the TB Diagnostic Cascade

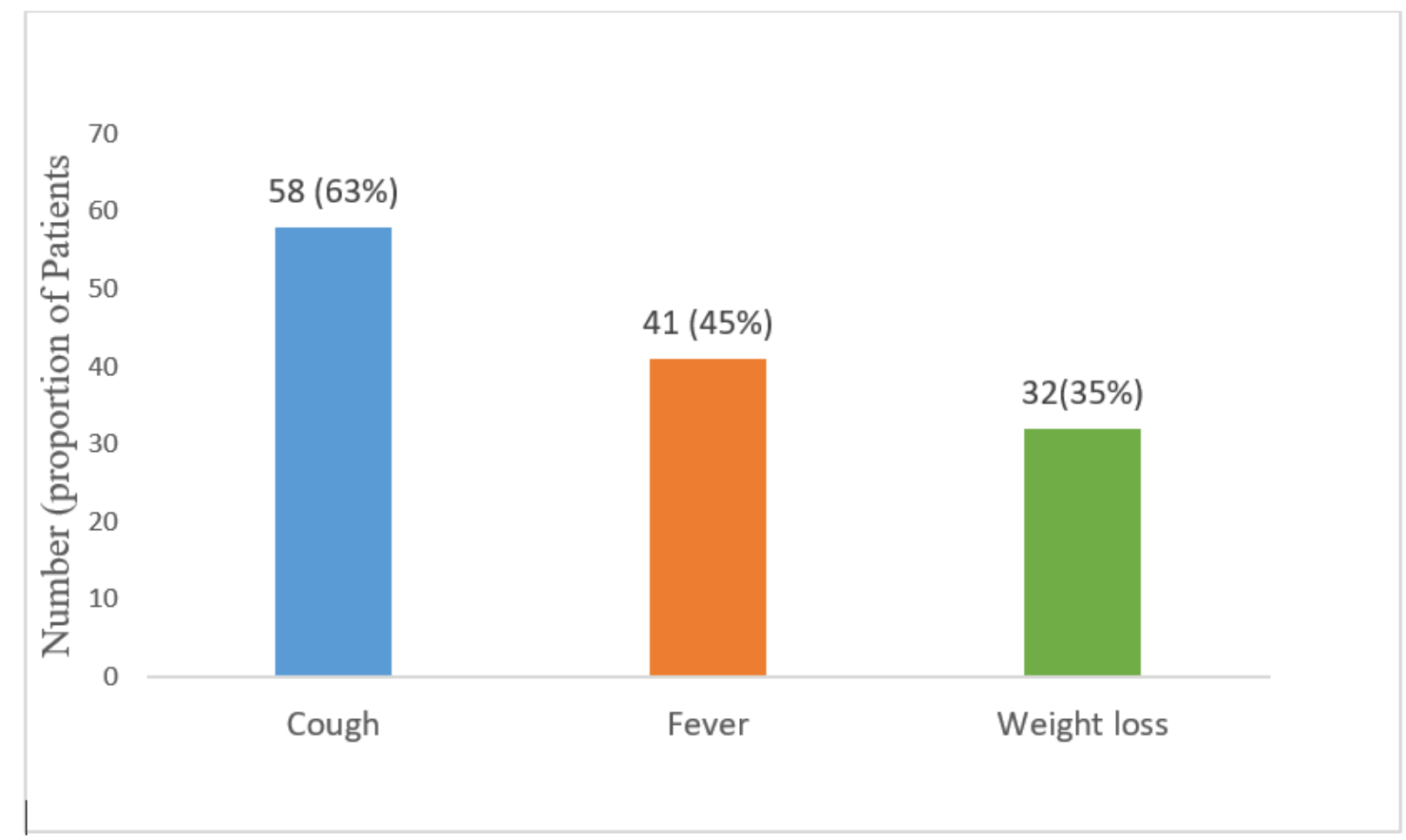

\section{Figure 2}

Distribution of TB symptoms in patients with a positive TB screen test, N=92 


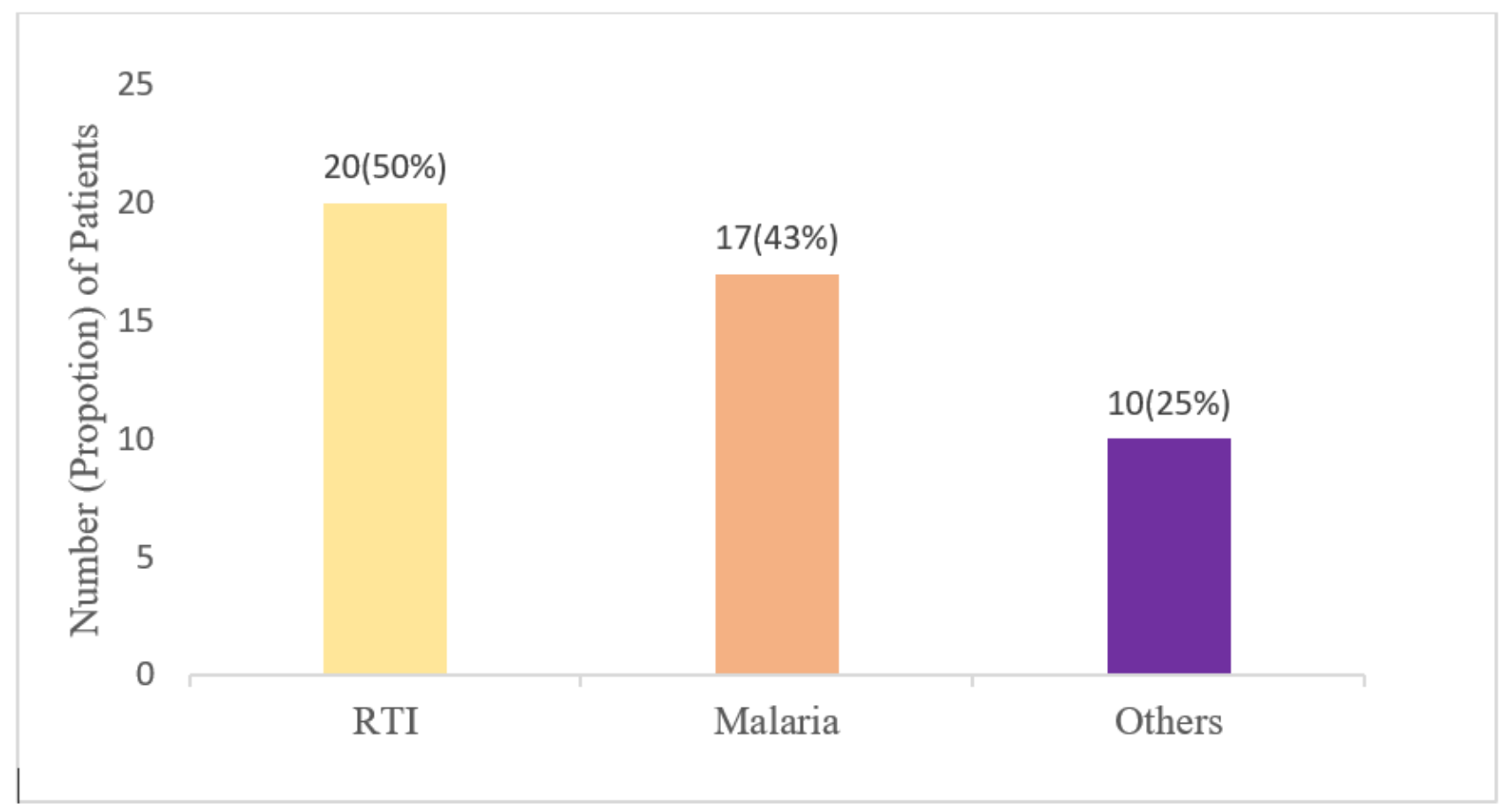

Figure 3

Common Alternative Diagnoses, N=40 